\title{
Pregnancy outcomes in cystic fibrosis: a I0-year experience from a UK centre
}

\author{
M Renton', L Priestley², L Bennett ${ }^{2}$, L Mackillop ${ }^{2}$ and \\ SJ Chapman ${ }^{2}$
}

SSAGE

\begin{abstract}
Background: Cystic fibrosis manifests as a multisystem disease, despite this female fertility is relatively preserved with levels approaching that of the non-cystic fibrosis population. We reviewed pregnancies in cystic fibrosis patients over a 10-year period from a UK adult cystic fibrosis centre by considering maternal and fetal outcomes.

Methods: We conducted a retrospective case-note review of pregnancies during 2003-2013 using respiratory and obstetric records.

Results: We observed moderate falls in lung function immediately after delivery, which persisted at 12 months postpartum. We found that a decline in lung function at delivery was a marker for further decline in function during the subsequent postpartum period. We found baseline lung function was predictive of gestational age at delivery. We observed a high incidence of haemoptysis.
\end{abstract}

Conclusion: Consistent with current guidance we found pregnancy is feasible and well tolerated in the majority of patients with cystic fibrosis. There was a high incidence of haemoptysis, which warrants further study.

\section{Keywords}

High-risk pregnancy, maternal-fetal medicine, thoracic medicine, complications, perinatal medicine

\section{Introduction}

Cystic fibrosis (CF) is the most common genetic condition in the Caucasian population. CF occurs due to mutations in the cystic fibrosis transmembrane conductance regulator (CFTR) gene. The CFTR ion channel facilitates sodium, chloride and bicarbonate transport, and functions as a regulator of surface liquid. ${ }^{1} \mathrm{CF}$ manifests as a multisystem disease involving the exocrine glands and epithelial tissues of the pancreas, sweat glands and mucous glands of the respiratory, digestive and reproductive tracts. ${ }^{2}$

Advancements in understanding and clinical management of $\mathrm{CF}$ have led to a progressive increase in life expectancy, with a predicted mean survival of greater than 50 years for CF patients born in the 21st century. ${ }^{3,4}$ Despite the multisystem effects of $\mathrm{CF}$, female fertility is relatively preserved with levels approaching that of the non-CF population. ${ }^{5,6}$ As a result pregnancy in CF patients is becoming increasingly relevant, with annual rates increasing dramatically. ${ }^{3,5,7}$ In general, outcomes reported in the setting of mild CF disease appear to be successful, and without long-term implications on maternal health or survival. ${ }^{8,9}$ Whilst pregnancy can be well-tolerated in CF patients, the additional physiological demands of pregnancy can however lead to deterioration in maternal condition with deleterious effects on maternal and fetal health. ${ }^{10}$

Guidelines advocate optimising preconception respiratory, cardiovascular and metabolic parameters. ${ }^{8}$ Poor lung function is commonly cited as the most significant single predictor of maternal morbidity, prematurity and fetal complications. ${ }^{11-20}$ Pregnancy is not recommended in significant lung disease (forced expiratory volume in one second (FEV1) $<50 \%$ predicted) with a low body mass index (BMI), whilst cor pulmonale and pulmonary hypertension are considered absolute contraindications. ${ }^{6,8,11}$ Current understanding and antenatal counseling is limited by a paucity of published series. The aim of the current study is to review pregnancies in CF patients over a 10 -year period from a UK adult CF centre by considering maternal and fetal outcomes.

\section{Methods}

We performed a clinical database search for all pregnancies occurring in patients with $\mathrm{CF}$ at Oxford University Hospitals NHS Trust, UK.
This facilitated a retrospective case-note review of all the pregnancies during the period 2003-2013 at this centre, using both respiratory and obstetric records.

Initial demographics considered were maternal age, BMI, pancreatic status, CF-related diabetes, Pseudomonas aeruginosa colonization, pulmonary function using FEV1 and forced vital capacity (FVC) during 6 months prior to pregnancy.

Longitudinal parameters and outcome measures were recorded for outcome of pregnancy, mode of delivery, gestational age at delivery, birth weight, obstetric cholestasis, gestational diabetes, haemoptysis, postnatal depression and pulmonary function at delivery, 6 and 12 months postpartum. All spirometry measures were best-recorded values. Haemoptysis was characterised as fresh bleeding outside the context of exacerbations, occurring during or after $(<12$ months) pregnancy.

Statistical analysis: descriptive statistics were analysed using SPSS Statistical Packages Social Sciences version 21. The FEV1 and FVC were calculated as percentage predicted values. Pearson's two-tailed correlations were performed, with a $p$ value of $<0.05$ considered statistically significant.

\section{Results}

Our clinical database search identified 15 singleton pregnancies in eight women. The descriptive statistics are summarized in Table 1. Prior to pregnancy, all women were offered usual adult $\mathrm{CF}$ care including at least 3-monthly review by a multidisciplinary team at the CF Centre; women attended for regular $\mathrm{CF}$ medical appointments preconception in 11 out of the 15 pregnancy cases. The combined oral contraceptive pill was used prior to pregnancy by six out of eight women; information on prior contraception in the remaining two women was not

\footnotetext{
'St John's College, University of Oxford, Oxford, UK

${ }^{2}$ Oxford University Hospitals NHS Trust, Oxford, UK
}

Corresponding author:

M Renton, St. John's College, St. Giles, Oxford, OXI3JP, UK.

Email: mary.renton@nhs.net 
Table I. Descriptive statistics.

\begin{tabular}{lcll}
\hline Baseline & Mean \pm SD & Range & \multicolumn{1}{l}{ Median } \\
\hline Age & $26.9 \pm 7.1$ & $17.0-38.0$ & 27.0 \\
BMI & $24.0 \pm 4.8$ & $13.8-29.9$ & 24.0 \\
FEV, \%pred & $68.10 \pm 16.5$ & $37.0-98.0$ & 70.0 \\
FVC \%pred & $84.13 \pm 19.8$ & $51.0-124.0$ & 81.9 \\
FEV $/$ FVC & $0.78 \pm 0.08$ & $0.64-0.93$ & 0.76 \\
Pancreatic insufficiency & & $11 / 15(73.3 \%)$ \\
CF-related diabetes & & $2 / 15(13.3 \%)$ \\
Pseudomonas aeruginosa colonization & & $13 / 15(86.7 \%)$ \\
\hline
\end{tabular}

BMI: body mass index; FEVI: forced expiratory volume; FVC: forced vital capacity.

available. All of the women conceived naturally with no requirement for assisted conception. Patients' vitamin A supplements were modified based on measurement of blood vitamin A levels, in line with UK guidelines on pregnancy in $\mathrm{CF}^{8}$ Baseline pulmonary function data were available for 11 out of 15 pregnancies. From this group, eight women had a baseline FEV1 $>60 \%$.

\section{Baseline cohort characteristics}

\section{Outcome measures}

Obstetric. Live births accounted for $13 / 15$ of the pregnancy outcomes, with $1 / 15$ stillbirth and $1 / 15$ therapeutic termination. Vaginal deliveries occurred in $7 / 13$ of the cases, with the remaining $6 / 13$ deliveries by caesarean section. Deliveries were classified as preterm $(<37$ weeks gestational age) for $8 / 15$, with a mean gestational age of 36.0 weeks $( \pm 3.78$ range $24-40)$. Birth weight mean was $2624.6 \mathrm{~g}$ ( \pm 940.3 range $964-3842)$

Maternal health complications. Obstetric cholestasis was reported in $1 / 15$ pregnancy. Three out of 15 pregnancies were associated with the development of gestational diabetes, with further two pregnancies occurring in women with underlying CF-related diabetes (Table 1); all cases of diabetes were managed with insulin therapy and ongoing highcalorie dietary intake, in keeping with UK guidelines. ${ }^{8}$ Haemoptysis was recorded in $6 / 15(40 \%)$ pregnancies $(5 / 8$ women $62.5 \%)$. The haemoptysis developed during the pregnancy or follow-up $(<12$ months), with the exception of one patient with pre-existing intermittent haemoptysis, which worsened during pregnancy.

One maternal death occurred from complications of $\mathrm{CF}$, at 9 months following a therapeutic termination at 18 -weeks gestation, for the indications of maternal pulmonary hypertension and excessive risk to the mother in continuing the pregnancy; this patient's BMI was 13.8 prepartum. Postnatal depression was observed in $3 / 8$ women.

\section{Longitudinal pulmonary function}

Longitudinal pulmonary function was available for nine out of 15 pregnancies (Table 2). Pulmonary function was reduced at delivery and 6 months postpartum. FVC returned to above baseline values at 12 months follow-up, but a reduction in FEV1 of $5.77 \%$ persisted at 12 months postpartum.

\section{Correlations}

Pearson's correlation identified significant positive correlations between baseline FEV1 and FVC with gestational age at delivery
Table 2. Peripartum pulmonary function in CF (mean $\pm S D)$.

\begin{tabular}{lllll}
\hline & \multicolumn{2}{l}{ Lung function } \\
\cline { 2 - 5 } & \multicolumn{4}{c}{ Postpartum \% changes } \\
\cline { 3 - 5 } & Baseline & Delivery & 6 months & 12 months \\
\hline FEVI \%pred & $68.10 \pm 16.5$ & $-5.31 \pm 13.3$ & $-4.52 \pm 10.2$ & $-5.77 \pm 6.8$ \\
FVC \%pred & $84.13 \pm 19.8$ & $-6.78 \pm 11.9$ & $-3.01 \pm 13.2$ & $2.37 \pm 11.8$ \\
FEVI/FVC \%pred & $78.38 \pm 8.8$ & $-3.73 \pm 7.9$ & $-4.45 \pm 7.5$ & $-3.90 \pm 14.7$
\end{tabular}

FEVI pred: predicted forced expiratory volume in one second; FVC pred: predicted forced vital capacity.

( $r=746, p=013$ and $r=859, p=003$, respectively), demonstrating that higher baseline pulmonary function was significantly associated with a longer gestation period. Gestational age at delivery was found to be associated with FEV1/FVC at 12 months $(r=950, p=004)$ : the longer the gestation period, the more preserved the FEV1/FVC at 12 months postpartum.

Reductions at delivery of both FEV1 and FVC were associated with greater reductions in FEV1 at 6 months postpartum $(r=765$, $\mathrm{p}=017$ and $\mathrm{r}=669, \mathrm{p}=049$, respectively). FVC reductions at 6 months were correlated with greater reductions in FEV1 and FVC at 12 months $(\mathrm{r}=844, \mathrm{p}=035, \mathrm{r}=825, \mathrm{p}=043)$.

\section{Discussion}

Here we report a single-centre retrospective study of pregnancy outcomes in $\mathrm{CF}$. Consistent with current guidance we found pregnancy to be feasible and well-tolerated in the majority of patients with CF. In keeping with other recent studies, ${ }^{14,16,21}$ we observed that pregnancy is occurring at an older age (mean 26.9 years) with lower lung function (mean FEV1 68.1\%) than noted in older studies. ${ }^{11}$ Indeed, pregnancy most commonly occurs in relatively mild $\mathrm{CF}$, with recent UK data showing that $58.1 \% \mathrm{CF}$ pregnancies occurred with FEV1 $>60 \%{ }^{3}$ We did however observe moderate falls in FEV1 immediately after delivery, which persisted at 12 months postpartum (Table 2). In comparison, annual declines in FEV1 of $0.65-1.65 \%$ have been reported in another UK Centre for comparable (non-pregnant) birth cohorts albeit at an earlier age (18-22 years). ${ }^{22}$ Although the lack of matching to our pregnant cases precludes definitive conclusions, it is likely that our observed rate of FEV1 decline postpartum is significantly greater than expected in non-pregnant patients. Not unexpectedly, we found that a decline in lung function at delivery was a marker for further decline in function during the subsequent postpartum period.

We found baseline FEV1 and FVC measures to be significantly associated with gestational age at delivery. Recent UK data also found FEV1 to be significantly associated with gestational age at delivery. ${ }^{21}$ We reported high rates of preterm $(46.7 \%)$ and CS $(46.15 \%)$ deliveries. This is in keeping with data from most other studies, ${ }^{11,12}$ although differs from a recent report from Burden et al. ${ }^{21}$ of $100 \%$ vaginal deliveries, reflecting the multifactorial indications for CS.

The frequent finding of haemoptysis (in five out of eight women) outside the usual setting of pulmonary exacerbations is noteworthy and has not been reported in previous studies. This may simply be a chance finding reflecting the relatively small size of our cohort, although it warrants further investigation in a larger population sample. Pregnancy-related haemoptysis has previously been described as a distinct entity. ${ }^{23}$ There are suggestions that the associated increased cardiac output and pregnancy-related hormonal changes may trigger abnormal bronchial vessel development, expansion of pulmonary arteriovenous malformations and recannalisation of vessels. ${ }^{23-25}$

In addition to the physiological impact of pregnancy in $\mathrm{CF}$, the psychosocial implications must also be considered. Although estimates 
vary, clinical prevalence of postnatal depression is reported as $13 \%{ }^{26}$ In our cohort we reported that $37.5 \%$ of our patients suffered with postnatal depression, reflecting the need for maximum support from a multidisciplinary CF team beyond management of physical disease.

Despite reports of successful pregnancies in women with FEV1 $<60 \%$ in the setting of $\mathrm{CF}^{27}$ current guidelines do not recommend pregnancy with an FEV $1<60 \%{ }^{8}$ Despite this, $19.7 \%$ pregnancies occur with FEV1 $<40 \%$, and $22.2 \%$ with FEV1 $40-60 \%{ }^{3}$ Pregnancy in one of our patients with severe disease and very low BMI resulted in therapeutic termination and subsequent maternal death 9 months later. This case is similar to a report from the recent French CF literature. ${ }^{28}$ It remains challenging to reliably predict adverse outcomes based on available maternal factors. There is an urgent need for data on women with more advanced disease to enable appropriate antenatal care and risk prediction.

Even with good outcomes, pregnancy in CF is associated with higher rates of exacerbation and hospitalization. ${ }^{29}$ The success of pregnancy is reliant on integrated care from a multidisciplinary team of CF specialists, obstetricians, geneticists, obstetric anaesthetists, dietitians and fetal medicine experts. With the continuing improvement in survival rates, pregnancy management in CF will increasingly become a routine part of care for many patients. Large, prospective studies of maternal and fetal outcomes are needed in order to inform optimal management of pregnancy in this patient group.

\section{Declaration of conflicting interests}

The authors declare there is no conflict of interest.

\section{Funding}

This research received no specific grant from any funding agency in the public, commercial or non-for-profit sectors. SJC is funded by the Medical Research Council UK and the NIHR Oxford Biomedical Research Centre.

\section{Ethical approval}

This study comprises a retrospective assessment of clinical course and outcome of anonymised patients who had received usual clinical care, without randomisation or experimental intervention, and hence did not require ethical approval or patient consent.

\section{Guarantor}

SJ Chapman.

\section{Contributorship}

MR and SJC conceived and designed the study. MR and LP performed data collection. MR performed data analysis. MR and SJC drafted the manuscript. All authors read and commented on the final manuscript.

\section{References}

1. Davies JC, Alton EWFW and Bush A. Cystic fibrosis. British Med J 2007; 335: 1255-1259.

2. O'Sullivan BP and Freedman SD. Cystic fibrosis. Lancet 2009; 13: 1891-1904.

3. Cystic Fibrosis Trust. UK CF Registry Annual Data Report, 2010.

4. Dodge JA, Lewis PA, Stanton M, et al. Cystic fibrosis mortality and survival in the UK: 1947-2003. Eur Respir J 2007; 29: 522-526.

5. Tonelli MR and Aitken ML. Pregnancy in cystic fibrosis. Curr Opin Pulm Med 2007; 13: 537-540.

6. Edenborough FP. Women with cystic fibrosis and their potential for reproduction. Thorax 2001; 56: 649-655.

7. Lemonnier L, Cazes MH, Bellis G, et al. WS18. 1 Evolution of pregnancies in the French CF Registry: 1992-2011. J Cystic Fibrosis 2013; 12: S36.
8. Edenborough FP, Borgo G, Knoop C, et al. Guidelines for the management of pregnancy in women with cystic fibrosis. $J$ Cystic Fibrosis 2008; 7: S2-S32.

9. Goss $\mathrm{CH}$, Rubenfeld GD, Otto K, et al. The effect of pregnancy on survival in women with cystic fibrosis. CHEST $J$ 2003; 124: $1460-1468$

10. Knight $\mathrm{M}$, Kenyon $\mathrm{S}$, Brocklehurst $\mathrm{P}$, et al. MBRRACEUK: Saving Lives, Improving Mothers' Care - Lessons learned to inform future maternity care from the UK and Ireland Confidential Enquiries into Maternal Deaths and Morbidity 200912. Oxford: National Perinatal Epidemiology Unit, University of Oxford, 2014.

11. Edenborough FP, Mackenzie WE and Stableforth DE.. The outcome of 72 pregnancies in 55 women with cystic fibrosis in the United Kingdom 1977-1996. BJOG 2000; 107: 254-261.

12. Thorpe-Beeston JG, Madge S, Gyi K, et al. The outcome of pregnancies in women with cystic fibrosis - single centre experience 1998-2011. BJOG 2013; 120: 354-361.

13. ØDegaard I, Stray-Pedersen B, Hallberg K, et al. Maternal and fetal morbidity in pregnancies of Norwegian and Swedish women with cystic fibrosis. Acta Obstetricia et Gynecologica Scand 2002; 81: 698-705.

14. Jankelson D, Robinson M, Parsons S, et al. Cystic fibrosis and pregnancy. Austr New Zealand J Obstetr Gynaecol 1998; 38: 180-184.

15. Gillet D, De Braekeleer M, Bellis G, et al. Cystic fibrosis and pregnancy. Report from French data1980-1999). BJOG 2000; 109: 912-918.

16. Lau EM, Barnes DJ, Moriarty C, et al. Pregnancy outcomes in the current era of cystic fibrosis care: a 15-year experience. Austr New Zealand J Obstetr Gynaecol 2011; 51: 220-224.

17. Etherington C, Peckham D, Conway S, et al. Pregnancy and motherhood in women with cystic fibrosis: experience and outcomes in a regional adult UK centre. J Cystic Fibrosis 2011; 384: S98.

18. Thorpe-Beeston JG.. Contraception and pregnancy in cystic fibrosis. JRSM 2009; 102: 3-10.

19. Lau EM, Moriarty C, Ogle R, et al. Pregnancy and cystic fibrosis. Paediatr Resp Rev 2010; 11: 90-94.

20. Whitty JE. Cystic fibrosis in pregnancy. Clin Obstetr Gynecol 2010; 53: 369-376.

21. Burden $\mathrm{C}$, Ion R, Chung $\mathrm{Y}$, et al. Current pregnancy outcomes in women with cystic fibrosis. Eur J Obstetr Gynecol Reprod Biol 2012; 164: 142-145.

22. Que C, Cullinan P and Geddes D. Improving rare of decline of FEV1 in young adults with cystic fibrosis. Thorax 2006; 61: $155-157$.

23. Peyrat E, Chabbert V, Escamilla R, et al. Idiopathic hemoptysis in pregnant women: A distinct entity? Resp Med 2007; 101: 2221-2223.

24. Weiss BM and Hess OM. Pulmonary vascular disease and pregnancy: current controversies, management strategies, and perspectives. Eur Heart $J$ 2000; 21: 104-115.

25. Jean-François De, Wispelaere MD, Trigaux JP, Weynants $P$, et al. Systemic supply to a pulmonary arteriovenous malformation: potential explanation for recurrence. Cardiovasc Interven Radiol 1996; 19: 285-287.

26. O'Hara M and Swain A. Rates and risk of postpartum depression: a meta-analysis. Int Rev Psychiatry 1996; 8: 37-54.

27. Canny GJ, Corey M, Livingstone RA, et al. Pregnancy and cystic fibrosis. Obstetr Gynecol 1991; 77: 850-853.

28. Guerci P, Vial F, Raft J, et al. Medical termination of pregnancy in a patient with severe cystic fibrosis. Possible effect of the antiglucocorticoid action of mifepristone on the respiratory disease. Ann Fr Anesth Rean 2013; 32: 115-117.

29. McMullen AH, Pasta DJ, Frederick PD, et al. Impact of pregnancy on women with cystic fibrosis. CHEST J 2006; 129: 706-711. 